\title{
An Analysis on Perturbation Features of Convection-Allowing Ensemble Prediction Based on the Local Breeding Growth Mode
}

\author{
Shenjia Ma, ${ }^{\mathrm{a}, \mathrm{b}, \mathrm{c}, \mathrm{g}}$ ChaOhui Chen, ${ }^{\mathrm{a}, \mathrm{c}, \mathrm{g}}$ Hongrang He, ${ }^{\mathrm{a}, \mathrm{c}}$ Jie XiAng, ${ }^{\mathrm{a}}$ ShengJie Chen, ${ }^{\mathrm{c}, \mathrm{d}}$ \\ Yi Li, ${ }^{\mathrm{a}}$ YONGQIANG JiAnG, ${ }^{\mathrm{a}}$ DAN Wu, ${ }^{\mathrm{a}, \mathrm{e}}$ AND HAO LUO ${ }^{\mathrm{f}}$ \\ ${ }^{a}$ College of Meteorology and Oceanography, National University of Defense Technology, \\ Nanjing, China \\ ${ }^{\mathrm{b}}$ PLA Troop 78127, Chengdu, China \\ ${ }^{\mathrm{c}}$ Nanjing Joint Center of Atmospheric Research, Nanjing, China \\ d Jiangsu Provincial Meteorological Observatory, Nanjing, China \\ ${ }^{\text {e }}$ PLA Troop 31631, Huizhou, China \\ ${ }^{\mathrm{f}}$ PLA Troop 95788, Chengdu, China
}

(Manuscript received 3 July 2018, in final form 8 November 2018)

\begin{abstract}
In this study, a convection-allowing ensemble prediction experiment was conducted on a strong convective weather process, based on the local breeding growth mode (LBGM) method proposed according to the strongly local nature of the convective-scale weather system. A comparative analysis of the evolution characteristics of the initial perturbation was also performed, considering the results from the traditional breeding growth mode (BGM) method, to enhance understanding and application of this new initial perturbation generation method. The experimental results showed that LBGM results in the perturbation distribution exhibiting characteristics more evident of flow dependence, and an initial perturbation with greater definite kinetic significance was derived. Information entropy theory could well measure the amount of information contained in the perturbation distribution, indicating that the innovative initial perturbation generation method can increase the amount of local information associated with the initial perturbation. With regard to the physical perturbation quantities, the LBGM method can improve the dispersion of the ensemble prediction system, thereby solving the problem of insufficient ensemble spread of prediction systems obtained by the traditional BGM method. Simultaneously, the root-mean-square error of the prediction can be further reduced, and the predicted precipitation distribution is closer to the observed precipitation, thereby improving the prediction effect of the convection-allowing ensemble prediction. The LBGM method has advantages compared to the traditional method and provides a new theoretical basis for further development of initial perturbation technologies for convection-allowing ensemble prediction.
\end{abstract}

\section{Introduction}

Evolving for more than half a century, ensemble prediction has successfully expanded from the global large-scale numerical model to the regional mesoscale numerical model (Zhang et al. 2017). Prediction centers around the world have also established their own regional ensemble prediction systems (Bentzien and

\footnotetext{
g Shenjia Ma and Chaohui Chen are co-first authors.
}

Corresponding author: Chaohui Chen, chenchaohui2001@ 163.com
Friederichs 2012; Wang et al. 2011, 2012; Zhang et al. 2015; Tennant 2015; Schwartz et al. 2015a; Weidle et al. 2016). As the demand for extreme weather prediction continues to increase, and the significant improvement in computing power satisfies the computing requirements of high-resolution prediction models, convection-allowing ensemble prediction has become a popular current research topic. At the same time, the ensemble prediction perturbation method, which is at the core of ensemble prediction study, has also received extensive attention from researchers all over the world. Typical perturbation methods include the Monte Carlo method (Leith 1974), the lagged average forecasting 
method (LAF; Hoffman and Kalnay 1983), the breeding growth mode (BGM; Toth and Kalnay 1993, 1997), the singular vectors method (SVs; Buizza and Palmer 1995), the perturbed observation method (PO; Houtekamer and Derome 1995), the ensemble transformation Kalman filter method (ETKF; Bishop et al. 2001; Wang and Bishop 2003), the ensemble transformation (ET; Wei et al. 2008), the conditional nonlinear optimal perturbation method (CNOP; Mu and Jiang 2008; Jiang et al. 2009), the ensemble Kalman filter method (EnKF; Jones and Stensrud 2012), and the nonlinear local Lyapunov vectors method (NLLV; Feng et al. 2014; Ding et al. 2017).

In recent years, the National Center for Atmospheric Research (NCAR) and the Center for Analysis and Prediction of Storms (CAPS) have conducted continuous convection-allowing ensemble prediction operational experiment and case studies, with the indicative function of precipitation and extreme weather as the focus of evaluation. They have also performed preliminary analysis on the trial results (Xue et al. 2007; Kong et al. 2008, 2009; Clark et al. 2012; Schwartz et al. 2014, 2015a,b). The results showed that convection-allowing ensemble prediction can improve the ability to identify precipitation intensity in a certain area and has guiding function for high-impact convective weather events. Zhuang et al. (2016) constructed a convection-allowing ensemble prediction system based on the interaction between the initial and lateral boundary perturbations of ETKF and dynamic downscaling. Subsequently, they conducted a case study of convection-allowing ensemble prediction for the Beijing " 7.21 " torrential rain (Zhuang et al. 2017). At present, the perturbation methods of a convection-allowing ensemble fall into three categories: the first is the use of downscaled members of a global ensemble prediction system as the initial condition perturbations and boundary conditions (Zhang et al. 2010; Peralta et al. 2012; Kühnlein et al. 2014), the second is the use of the final analysis results from multiple prediction centers as the initial condition perturbations (Arribas 2005; Bowler et al. 2008), and the third is the generation of multiscale initial condition perturbations by a cycled ensemblebased data assimilation system (Harnisch and Keil 2015; Johnson and Wang 2016). In general, the dominant approach is to use global orthogonality to consider the initial condition perturbations, but this has some disadvantages (Chen et al. 2018). Taking ETKF as an example, on the one hand, it needs highly stable and accurate observation systems as support. On the other hand, all physical variables are included in the same vector when ETKF transforms forecast perturbations into analysis perturbations. As the physical variables are different in physical significances and orders of magnitude, the rationality and accuracy of such transformation remain to be further studied.

Considering that BGM is a popular initial perturbation method, the same as in ET and ETKF, it obtains the fastest growth perturbation by the dynamic cycling. However, the perturbations generated by ET and ETKF are given by the analysis error standard covariance matrix input by the data assimilation system, while the BGM method breeds the initial perturbations through the short-term prediction cycle of the model itself and does not require additional observation data. Gao et al. (2010) designed a convection-allowing ensemble prediction trial for supercell storms of the United States, which preliminarily validated the rationality and value of the BGM method in convection-allowing ensemble prediction. Li et al. (2017) and Ma et al. (2018a,b) have conducted convection-allowing ensemble prediction trials for a squall-line system. The results of deterministic prediction, probabilistic prediction, and scoring verifications all preliminarily indicated that the BGM method is effective for convection-allowing ensemble prediction.

However, Pena and Kalnay (2004) pointed out that the BGM method with a 6-hourly interval can only breed synoptic perturbation modes. And the main drawback of this method is that the dispersion of the ensemble prediction system is not large enough, which is an important factor restricting the further development of the BGM method in high-resolution ensemble prediction. As a result, Chen et al. (2018) proposed the local breeding growth mode (LBGM), an innovative perturbation method that can, to some extent, improve the dispersion of the convection-allowing ensemble prediction system from a case study. This method, based on the BGM, attempts to obtain smaller-scale perturbations by local adjustment during the breeding phase. As an innovative perturbation method, the study of the evolution and development of perturbation is the basic work for conducting ensemble prediction, and it is also the key link in ensemble prediction. One of the first to apply the traditional BGM method, Toth and Kalnay (1993) analyzed the perturbations in the growth cycle and proved that the BGM method itself can select the initial perturbations, causing the rapidly growing perturbations to dominate. Yu and Zhang (2005) and Zhang and Luo (2009) constructed initial perturbations based on the BGM method and conducted saturation analysis of the growth rate and morphology of the perturbations. The results showed that the 
evolution characteristics of perturbations are closely related to the structure of the circulation background field. Magnusson et al. (2008) applied orthogonal and nonorthogonal breeding vector (BV; similar to BGM) methods to the low-order dynamic system Lorenz-63 model to study the initial perturbations. The results showed that the orthogonal perturbation method has the advantage of selecting local rapidly growing perturbations, and this conclusion has been validated by the ensemble prediction systems in the European Centre for Medium-Range Weather Forecasts.

In this study, based on the traditional BGM and innovative LBGM ensemble prediction systems, convection-allowing ensemble prediction experiments were conducted for strong convective weather processes in the Yangtze-Huaihe region. Information entropy of information theory was used to measure the amount of information contained in perturbation variables. The paper provides two objectives. The first one is, while focusing on the morphological distribution, information entropy, and ensemble spread, the evolution characteristics of perturbations in the growth phase are compared and analyzed to provide assessment and verification of LBGM put forward by Chen et al. (2018) and to provide a better understanding of it. The second one is that the comparison and analysis of observed and predicted precipitation prove that the new LBGM perturbation method is superior. Section 2 introduces the initial perturbation methods of LBGM, the related forcing data and analysis data. Section 3 describes the convective-scale weather case and the design of the experiment. The evolution characteristics of perturbations in the growth phase are examined in section 4, together with comparison and analysis of the results in the prediction phase. Finally, the conclusions are provided in section 5 .

\section{Data and methods}

In the traditional BGM method, after every 6-h growth cycle, the scale of the current perturbation field is adjusted dynamically to maintain the same magnitude as that of the initial perturbation with respect to the root-mean-square error (RMSE). The dynamic adjustment method is

$$
d f_{t}^{\prime}(k)=\frac{e_{0}(k)}{e_{t}(k)} d f_{t}(k),
$$

where $k$ denotes the index in the vertical layer, $t$ denotes the current moment of the growth cycle, $d f_{t}(k)$ is the perturbation after one growth cycle, $e_{t}(k)$ is the RMSE at the corresponding time point, $e_{0}(k)$ is the RMSE at the initial time point, and $d f_{t}^{\prime}(k)$ is the perturbation at the next growth time point after dynamic adjustment. The detailed calculation procedure is described in Li et al. (2017) and Ma et al. (2018b). The perturbation obtained by the classical BGM method is only a function of $k$. That is, only the heterogeneity of the physical perturbation quantities in the vertical direction is considered, while the perturbation throughout the same layer is equal, due to a global adjustment.

Because of the strongly local nature and nonlinear characteristics of convective-scale weather systems, the interaction among model grids cannot be ignored. Therefore, the global adjustment of the traditional BGM method limits the further development of convection-allowing ensemble prediction, and it is also an important cause of the insufficient ensemble spread. Consequently, in the proposed LBGM perturbation method, the local nature and independence of the physical perturbation quantities of convection-allowing ensemble prediction are taken into account, and a local adjustment method is adopted in place of the global adjustment in the traditional BGM method. The method of local adjustment is

$$
d f_{t}^{\prime}(i, j, k)=\frac{e_{0}(k)}{e_{t}(i, j, k)} d f_{t}(i, j, k) .
$$

The $i, j$ denote the index in latitude and longitude, and other symbols are the same as those in Eq. (1). The difference is that the perturbation obtained by the LBGM method is not only a function of $k$ but actually a function of $(i, j, k)$. That is, the heterogeneity of the physical perturbation quantities in the horizontal and vertical three-dimensional directions is also considered. This local adjustment is not only consistent with the initial perturbation in terms of the RMSE but also reflects the local characteristics of the convective-scale weather system.

The core of the implementation of the LBGM method is that local characteristics are taken into account in the RMSE of the 6-h prediction. The calculation formula is as follows:

$$
\begin{aligned}
& \operatorname{RMSE}(i, j, k) \\
& =\frac{1}{(2 r+1)^{2}} \sqrt{\sum_{i-r}^{i+r} \sum_{j-r}^{j+r}\left[X_{r}(i, j, k)-X_{\mathrm{ctl}}(i, j, k)\right]^{2}},
\end{aligned}
$$


Traditional BGM (whole domain)

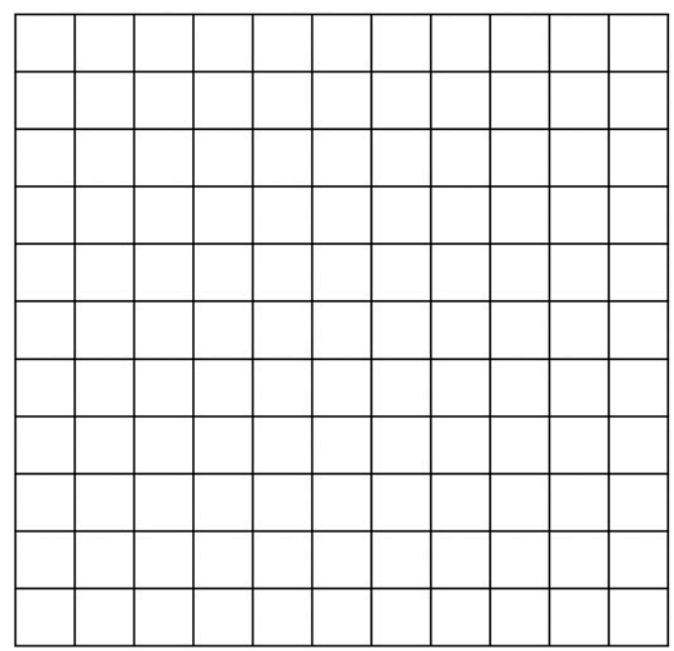

Local BGM ( $r=2$ )

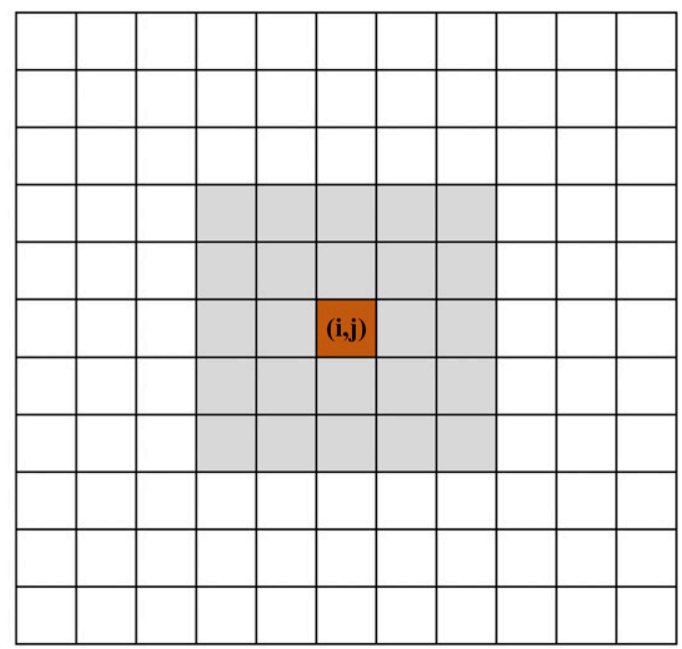

FIG. 1. Comparison between BGM and LBGM in deriving RMSE.

where $r$ represents the local influence radius, which is used to determine the number of local spatial grids; and $X_{r}(i, j, k)$ and $X_{\mathrm{ctl}}(i, j, k)$ represent the disturbance prediction field of the disturbance variable and the control prediction field of no addition of disturbance, respectively. Figure 1 shows a comparison between traditional BGM and LBGM methods in deriving the RMSE. In the traditional BGM method, the RMSE of a certain vertical level is calculated from all grids in the study area (as shown on the left side in Fig. 1). In the LBGM method, the RMSE of the brown grid cell $(i, j)$ in the graph is jointly determined by $(2 r+1)^{2}-1$ surrounding shadow grids (on the right side in Fig. 1, $r=2$ ). Therefore, at the same level, the RMSEs of all grids in the traditional BGM method are equal, while the RMSEs of all grids in the LBGM method are different and have local characteristics.

In the LBGM method, the perturbation of each grid cell is determined by its RMSE within the local influence radius, so that the locally adjusted perturbation includes the local information of the convective-scale weather system. This perturbation generation method, which takes into account the local characteristics, is similar to the neighborhood assessment method of Ebert (2008), Roberts and Lean (2008), Weusthoff et al. (2010), and Ma et al. (2018a), which takes into account the error in the precipitation area. Therefore, the conclusion derived by the postprocessing method based on neighborhood can be used as a reference. And Chen et al. (2018) conducted a preliminary sensitivity test on the local influence radius. The experiments show that 7-13 grids of influence radius can effectively increase the ensemble dispersion of the forecast system while decreasing the RMSE, making the forecast more stable and functional. As such, the authors referred to the relevant conclusions of $\mathrm{Ma}$ et al. (2018a) from the precipitation spatial-scale assessment for convectionallowing ensemble prediction and Chen et al. (2018) from the sensitivity test on the radius in LBGM method, and adopted the LBGM results with a local influence radius of $r=13$ to compare with the global BGM results.

NECP FNL reanalysis data were adopted for the model and weather analysis. The China Hourly Merged Precipitation Analysis $0.1^{\circ} \times 0.1^{\circ}$ grid data from the National Meteorological Information Center (NMIC) were used as the observed precipitation field for comparison.

\section{Case studies and designs}

From the afternoon of 30 July 2014 to the early morning of 31 July 2014, the Yangtze-Huaihe region in China experienced a strong large-scale convective weather process. Affected by this weather process, Henan, Anhui, Jiangsu, and other areas suffered severe convective weather, such as thunderstorms and strong winds, which caused damage and collapse of a large number of houses and severe urban water logging. From 0600 to 1100 UTC 30 July, an east-west strong squall line swept from north to south across the central and northern regions of the Jiangsu and Anhui Provinces. In Mingguang City, winds above $14 \mathrm{~m} \mathrm{~s}^{-1}$ occurred, and heavy rainfall occurred in some areas of Changfeng Town. The rainfall reached $75 \mathrm{~mm}$ in one hour.

Through the analysis of the upper-level synoptic situation, the trough line at 200-hPa level (figure omitted) 

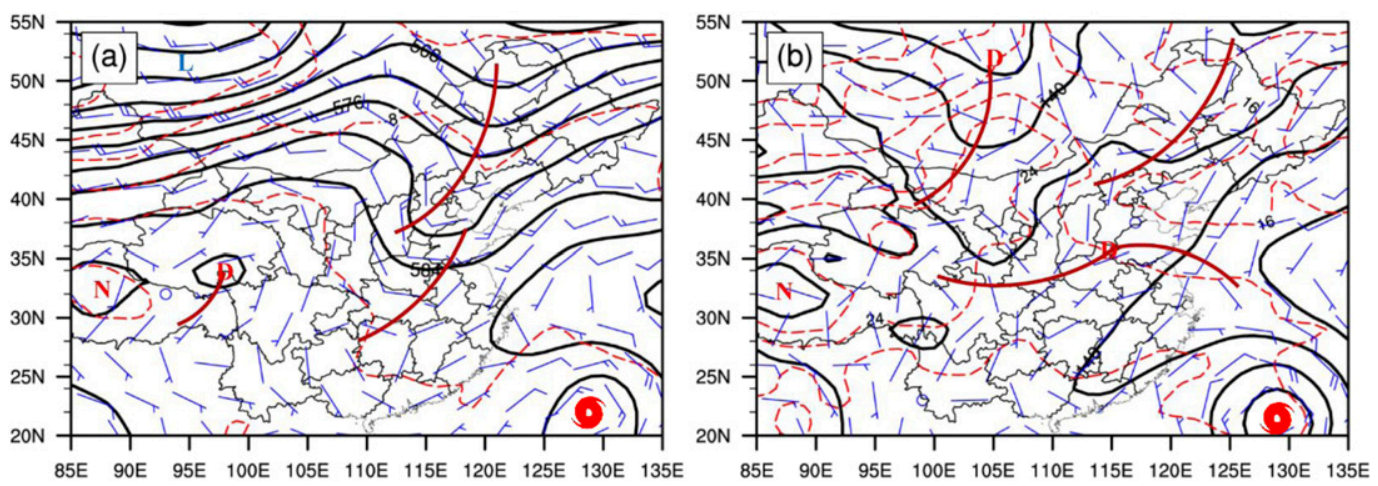

FIG. 2. The isobaric charts at 0000 UTC $30 \mathrm{Jul}$ 2014: (a) 500 and (b) $850 \mathrm{hPa}$. Black solid lines are geopotential height $(\mathrm{gpm})$, and red dashed lines are isotherms $\left({ }^{\circ} \mathrm{C}\right)$.

was between western Daxing'anling and Hebei Province, behind which is a cold center at the east of Lake Baikal with the lowest temperature below $-56^{\circ} \mathrm{C}$. There is a northwest upper-level jet stream behind the trough line and a southwest upper-level jet stream in front of it. Under the guidance of the two jet streams, the trough line moves quickly eastward, creating a suitable flow field for cold air heading toward northern China. The trough line at the 500-hPa level (Fig. 2a) spilt into two above northern China and formed a step-shape situation. Behind the northern trough line, there is high speed wind and cold advection. Yet the southern trough line has weak cold advection. Influenced by Typhoon Nakri, western Pacific subtropical high pressure is developing northwestward, slowing down the speed of the southern trough line and facilitating the step-shape trough lines to incorporate. Strong convective weather is highly possible under this circumstance. Above northern China at $850 \mathrm{hPa}$ (Fig. 2b) is a warm shear line. The southern-sided wind has higher speed, causing the warm, wet air from above the sea to accumulate around the shear line. In conclusion, this squall line is formed under the guiding of the stepshape trough line, which led the dry, cold air to south and overlying over the warm, wet air at the lower levels. The triggering mechanism is the warm shear line in front of the cold vortex.

In the experiment, a nonhydrostatic mesoscale model WRF v3.6 was adopted, and the model was set as a bidirectional triple-nested scheme. The regional settings are shown in Fig. 3. The resolution of the nested grids is $18 \mathrm{~km} \times 6 \mathrm{~km} \times 2 \mathrm{~km}$. The center of the parent grid cell is at $35^{\circ} \mathrm{N}, 115^{\circ} \mathrm{E}$, and its number of grid cells is $175 \times$ 175. The number of grid cells in the secondary grid $\mathrm{d} 02$ is $271 \times 271$. The number of grid cells in the tertiary grid $\mathrm{d} 03$ is $451 \times 451$. The number of vertical layers is 42 . In the perturbation growth stage, the traditional BGM method and the new LBGM method were considered for cocirculating growth for three days. From 0000 UTC 27 July to 0000 UTC 30 July 2014, with growth cycles of $6 \mathrm{~h}, 10$ ensemble members were set. The perturbation variables were the horizontal zonal wind speed $U$, the horizontal meridional wind speed $V$, the perturbation potential temperature $T$, the perturbation geopotential height $(\mathrm{PH})$, and the water vapor mixing ratio $Q$. The prediction period was from 0000 UTC 30 July to 0000 UTC 31 July for a total of $24 \mathrm{~h}$. At the same time, the two sets of ensemble prediction systems used the same physical parameterization scheme (as shown in Table 1). Therefore, the difference between the perturbation development and prediction results is only caused by the different perturbation adjustments of the two growth methods. It is possible to reasonably discuss the effects and values of the new LBGM method for convection-allowing ensemble prediction. Because of

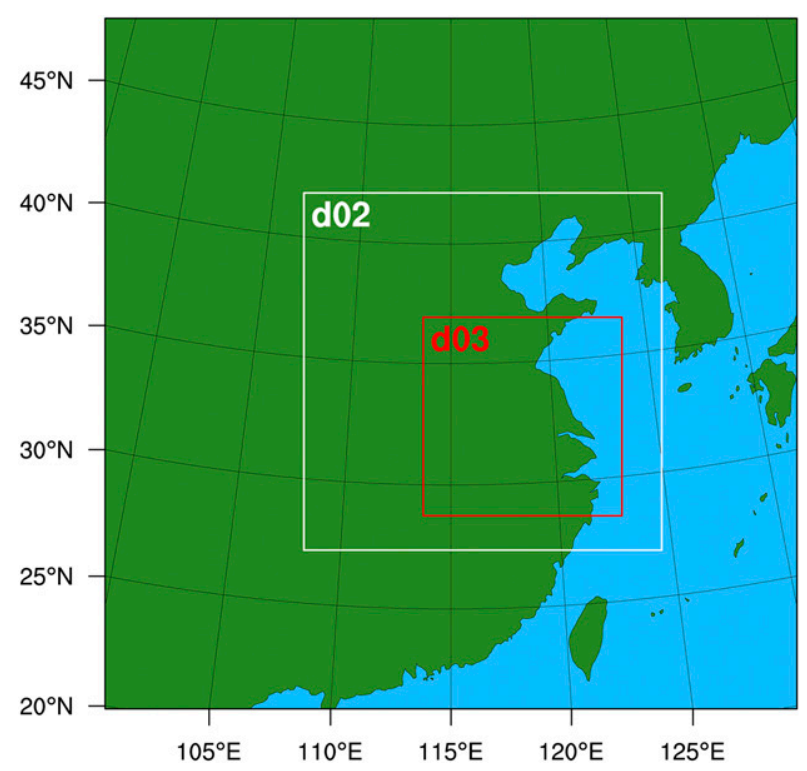

FIG. 3. WRF model area settings. 
TABLE 1. Basic physical parameterization scheme settings.

\begin{tabular}{ll}
\hline \multicolumn{1}{c}{$\begin{array}{c}\text { Parameterization } \\
\text { scheme }\end{array}$} & \multicolumn{1}{c}{ Prediction area } \\
\hline Cumulus & $\begin{array}{c}\text { Betts-Miller-Janjić scheme (area D03 } \\
\text { was excluded) } \\
\text { Morrison double-moment point scheme } \\
\text { Microphysics } \\
\text { Longwave radiation } \\
\text { Raphediative Transfer Model } \\
\text { scheme (RRTM) }\end{array}$ \\
$\begin{array}{l}\text { Surface layer } \\
\text { Land surface } \\
\text { Planetary boundary } \\
\text { layer }\end{array}$ & $\begin{array}{l}\text { Monin scheme } \\
\text { Noah land surface model }\end{array}$ \\
\hline
\end{tabular}

the errors introduced by the interpolation of observed data, in the forecasting phase, we referred to the perfect model assumption of Johnson and Wang (2016) and compared the forecasts of two perturbation methods with the control prediction as the true value.

\section{Results}

\section{a. Perturbation morphology distribution}

Figure 4 shows the evolution of the perturbation morphology distribution of the horizontal zonal wind field $U$ at the $850-\mathrm{hPa}$ level during perturbation growth. Comparing the distribution of the perturbation morphology before and after local adjustment, we can see that the perturbation distribution in the next growth cycle after adjustment provides more local information. [The perturbation before adjustment is $d f_{t}(i, j, k)$ in Eq. (2), and the perturbation at the corresponding time after adjustment is $d f_{t}^{\prime}(i, j, k)$ in Eq. (2).] Figures $4 \mathrm{a}, 4 \mathrm{c}$, and $4 \mathrm{e}$ show that the perturbation field before adjustment is relatively uniform, and the spatial scale of the positive and negative perturbation centers is large, which is insufficient to describe the strongly local characteristics of convective-scale weather systems. In the adjusted versions in Figs. $4 \mathrm{~b}, 4 \mathrm{~d}$, and $4 \mathrm{f}$, the spatial scale of each perturbation center is reduced, and more obvious local distribution characteristics are obtained. Taking the elliptic regions marked in Figs. 4e and $4 \mathrm{f}$ as examples, the former is an east-west westerly perturbation center, while after adjustment, seven small-scale westerly perturbation centers appear. At the same time, the perturbation distribution obtained after adjustment not only provides more local information but also maintains the basic structure of the perturbation distribution before adjustment. A similar phenomenon can be observed for the morphological evolution distribution of the remaining perturbation variables (figure omitted). The magnitude of the perturbation demonstrates that the adjusted perturbation size is stable (Figs. 4b,d,f), which can guarantee the
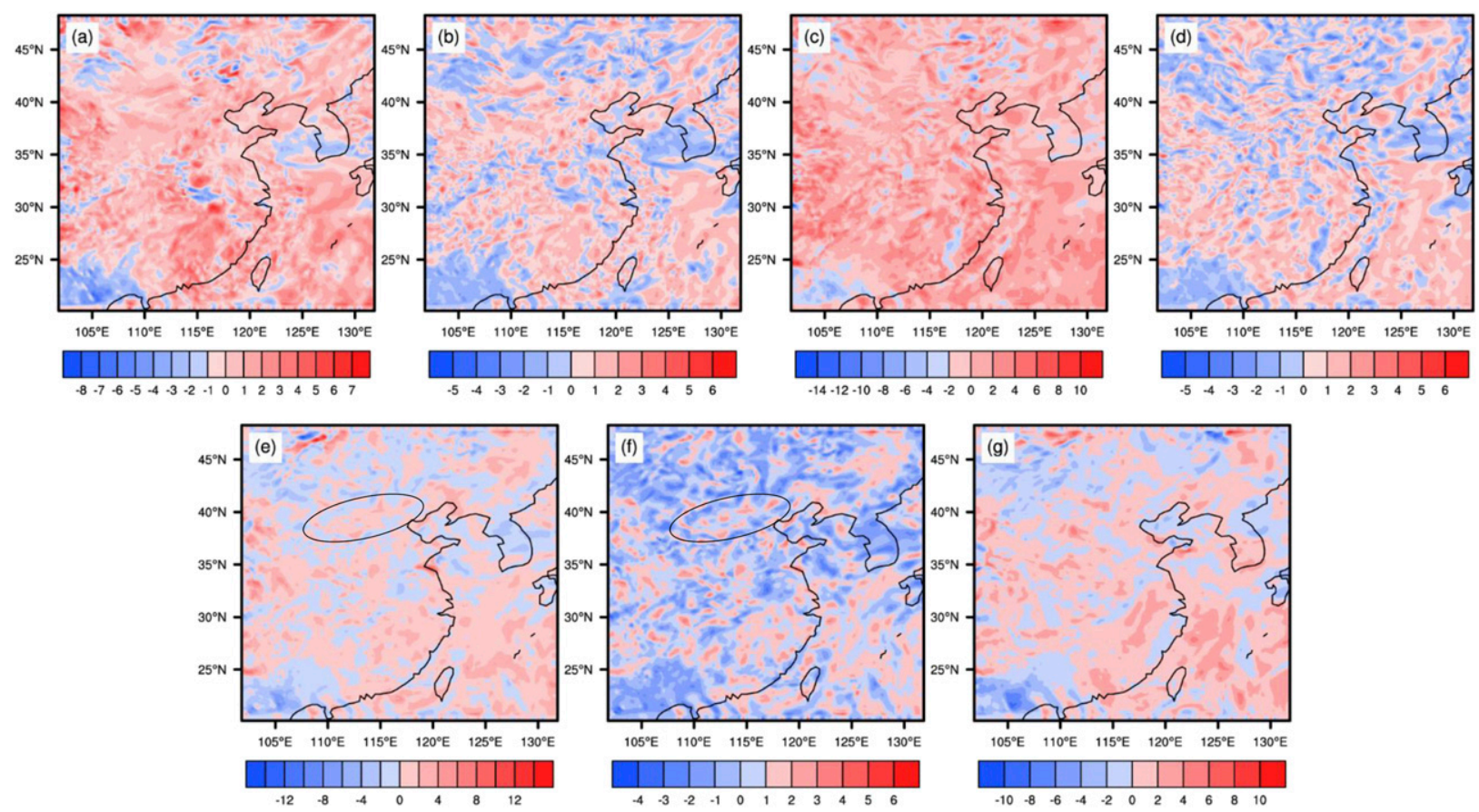

FIG. 4. Perturbation morphology distribution of the horizontal zonal wind field $U\left(\mathrm{~m} \mathrm{~s}^{-1}\right)$ at $850 \mathrm{hPa}$. The perturbation distributions before and after the local adjustment on the 29th day at (a),(b) 0600 UTC; (c),(d) 1200 UTC; and (e),(f) 1800 UTC. (g) The final perturbation field obtained at 0000 UTC on the 30th day. 

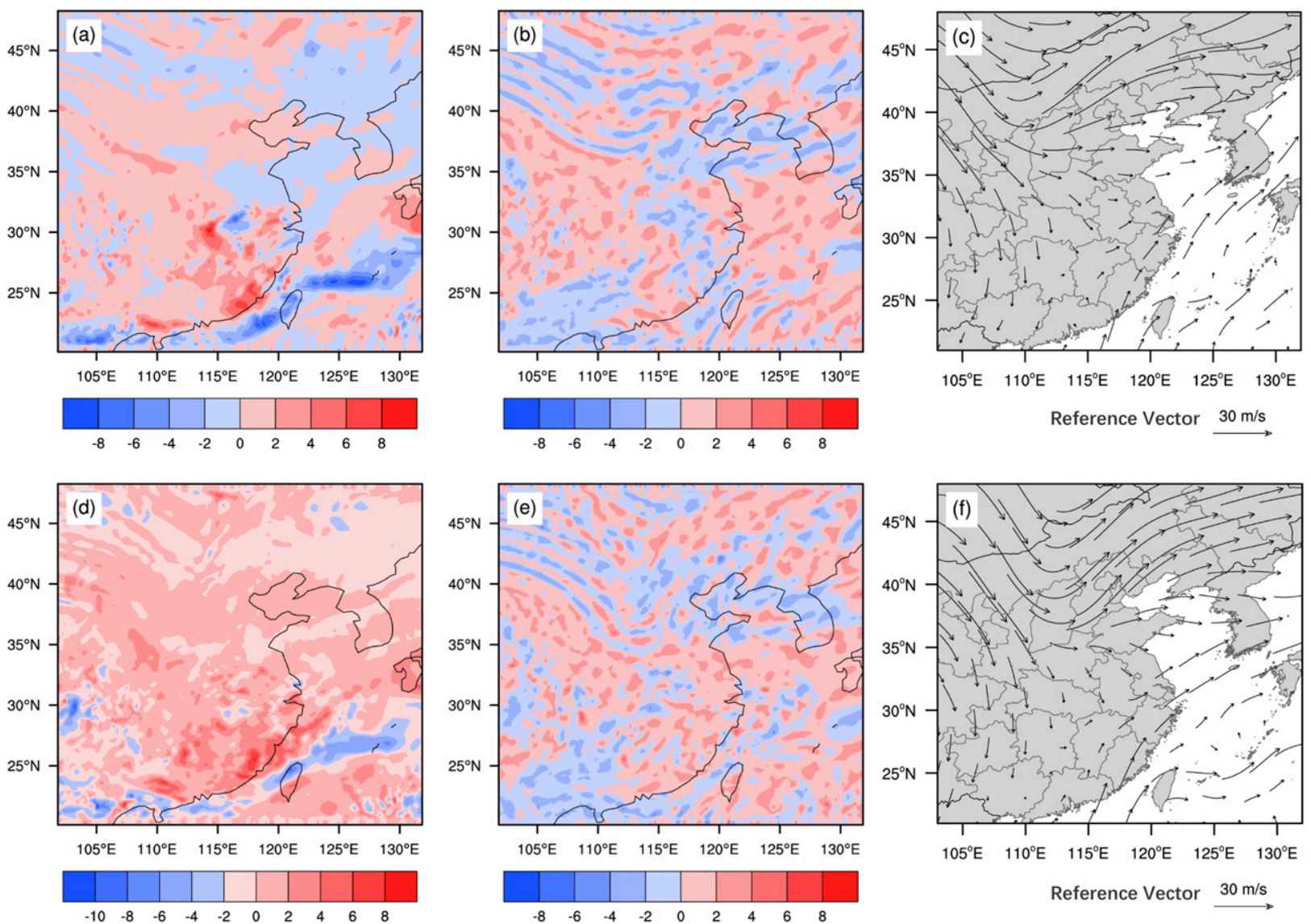

FIG. 5. Distributions of the horizontal wind field $\left(\mathrm{m} \mathrm{s}^{-1}\right)$ at $200 \mathrm{hPa}$. (a) Perturbation distribution after adjustment of the BGM method at 0600 UTC on the 29th day. (b) Perturbation distribution after adjustment of the LBGM method at 0600 UTC on the 29th day. (c) Analysis field at 0600 UTC on the 29th day. (d) Perturbation distribution after adjustment of the BGM method at 1200 UTC on the 29th day. (e) Perturbation distribution after adjustment of the LBGM method at 1200 UTC on the 29th day. (f) Analysis field at 1200 UTC on the 29th day.

rationality of the perturbation magnitude. This continues the advantages of scale adjustment for each growth cycle in the traditional BGM method. However, the BGM method only adjusts the global scale, while the structure of the perturbation distribution does not change. Therefore, the above analyses show that the local adjustment by the LBGM method not only ensures the overall structure of the perturbation distribution but also obtains more local perturbations and increases the amount of local information of the convective-scale weather system. The resulting perturbation field (Fig. 4g) reflects both the characteristics of the large-scale circulation background and the local interaction characteristics of the convective-scale weather system.

Figure 5 shows the perturbation distribution of the horizontal zonal wind field $U$ at $200 \mathrm{hPa}$ after adjustment by the BGM method and the LBGM method at 0600 and 1200 UTC on the 29th day, along with the corresponding reanalysis data wind field. (The website of data source is https://rda.ucar.edu/datasets/ds083.2/ index.html.) Compared to lower-layer adjustments, the higher-layer adjustments are basically not affected by near-surface factors, and the perturbation distribution scale obtained is larger. The perturbation distribution (Figs. 5b,e) after localized adjustment in the LBGM method not only maintains the basic structure of the perturbation obtained by the global adjustment of the traditional BGM method (Figs. 5a,d) but also has more perturbation information, causing the basic flow pattern of the perturbation distribution obtained by the LBGM method to be consistent with the wind field distribution at the corresponding time point (Figs. 5c,f). The comparison shows that compared to the traditional BGM method, the perturbation distribution obtained by the LBGM method exhibits characteristics more evident of flow dependence. Therefore, the local adjustment of the LBGM method has greater explicit 
kinetic significance than the global adjustment of the traditional BGM method.

\section{b. Comparative analysis on information entropy}

Shannon (1948) introduced the concept of thermodynamic entropy into the field of information theory and measured the uncertainty or amount of information (i.e., information entropy) contained in random variables through probability distribution, laying the scientific foundation for modern information theory. With the development of information theory, information entropy has been applied to the studies of predictability theory in the field of meteorology (Abramov et al. 2005; DelSole 2005), but most of the studies are limited to the qualitative study of the predictability of the prediction system ( $\mathrm{Li}$ et al. 2013).

Based on the information entropy of information theory, this study quantitatively analyzed the perturbation information amount of the traditional BGM and new LBGM prediction systems. The calculation formula is as follows:

$$
E(t)=-\int_{R^{n}} P[\mathbf{x}(t)] \times \log _{2}\{P[\mathbf{x}(t)]\} d \mathbf{x},
$$

where $\mathbf{x}(t)=\left[x_{1}(t), x_{2}(t), \ldots, x_{n}(t)\right]^{\mathrm{T}}$ is the perturbation variable of the prediction system at time point $t$, and $P[\mathbf{x}(t)]$ is the probability density function of the perturbation variable at time point $t$. The term $R^{n}$ represents the perturbation ensemble of the prediction system, and $E(t)$ is the information entropy at this time point (with 2 as the base of the logarithm and bit as the unit), which is used to measure the heterogeneity of the distribution field of the perturbation variable (i.e., to quantify the local information characteristics of the convective-scale weather system).

Figure 6 shows the evolution of information entropy of the perturbation morphology at the $850-\mathrm{hPa}$ level in the horizontal zonal wind field $U$ during perturbation growth. The dotted line in the figure shows that the information entropy before and after the global adjustment of the perturbation field at each time point in the traditional BGM method remains unchanged, which further shows that the global adjustment of the BGM method can only ensure the rationality of the perturbation magnitude and does not change the structure of the perturbation distribution. From the information entropy evolution of the perturbation distribution, the BGM method does not effectively increase the local information characteristics of the perturbation. From the solid line in the figure, there is a "jump" phenomenon in the information entropy before and after local adjustment of the perturbation field at each time point in the LBGM method, indicating that the

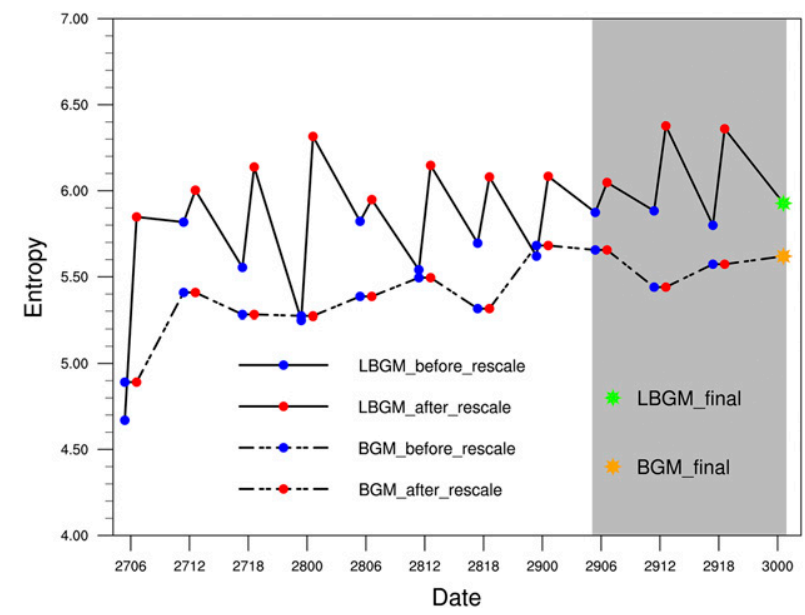

FIG. 6. Evolution of information entropy of the perturbation morphology at $850 \mathrm{hPa}$ in horizontal zonal wind field $U$. The solid line is the result obtained by the LBGM method, and the dashed line is the result obtained by the BGM method. The blue dots indicate the information entropy of the perturbation before adjustment, the red dots indicate the information entropy of the perturbation after adjustment, the green and yellow dots indicate the information entropy of the final perturbation obtained by the LBGM method and the BGM method, respectively, and the shaded part corresponds to the information entropy of the perturbation morphology distribution in Fig. 4.

local adjustment of the LBGM method can significantly increase the local information characteristics of the perturbation. Comparing the evolution of information entropy in the two methods shows that the information entropy of LBGM is higher than that of BGM. At the same time, the shaded part corresponds to the perturbation morphology distribution shown in Fig. 4. The visual image and the quantitative data demonstrate that the LBGM method can more effectively capture the local information of the convectivescale weather system than the BGM method.

Figure 7 shows the evolution of information entropy of the perturbation distribution of each perturbation variable after adjustment (entered into the prediction system), demonstrating that the information entropy values of the perturbation variables of the two methods are all equal at the initial time point of growth. This is because the perturbation distribution field at the initial time is initially entered into the system, when no global or local adjustments have been made. The perturbation fields obtained by the two methods have the same structure. In particular, the adjusted perturbation field is the perturbation input field, so the perturbation input field at the initial time point is not adjusted. However, as demonstrated in Fig. 6, to compare the information entropy evolution of the perturbation distribution before and after adjustment, the information entropy of 

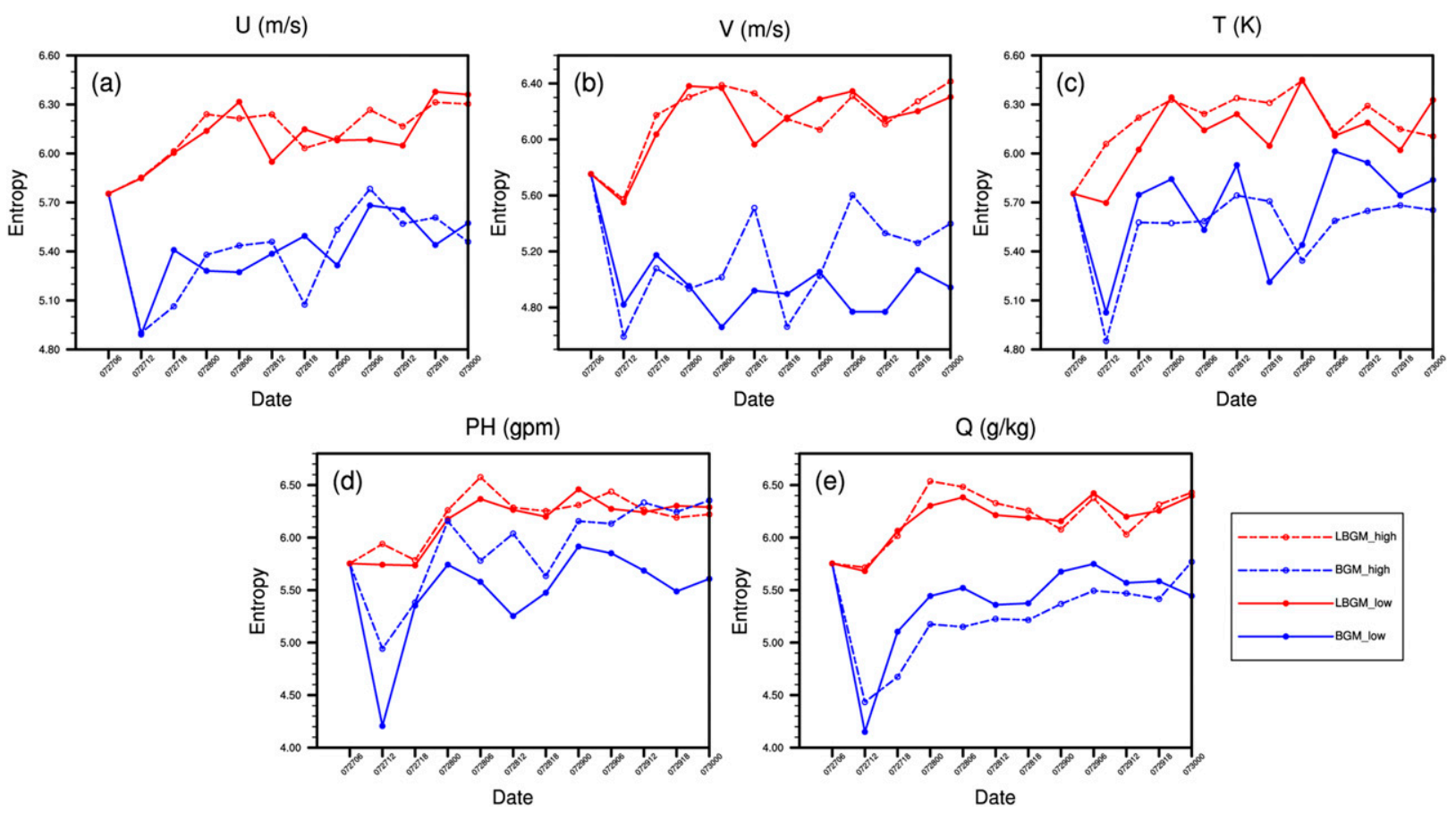

FIG. 7. Evolution of the information entropy after adjustment of the perturbation variables in the growth phase. (a) The horizontal zonal wind field $U\left(\mathrm{~m} \mathrm{~s}^{-1}\right)$, (b) horizontal meridional wind field $V\left(\mathrm{~m} \mathrm{~s}^{-1}\right)$, (c) perturbation potential temperature $T(\mathrm{~K})$, (d) perturbation geopotential height ( $\mathrm{PH}$; gpm), and (e) water vapor mixing ratio $Q\left(\mathrm{~g} \mathrm{~kg}^{-1}\right)$. The blue line and the red line are the results of the traditional BGM method and the LBGM method, respectively. The solid line represents the 850-hPa level, and the dashed line represents the 500-hPa level.

the perturbation input field at the initial time point is withheld, resulting in the information entropy of the perturbation variable $U$ in the two methods at the initial time point not being equal. At the same time, whether at the lower or higher layers, the information entropy of each perturbation variable obtained by the LBGM method is greater than the traditional BGM method (the red line is above the blue line). Therefore, the LBGM method can significantly increase the amount of information of the initial perturbation compared with the traditional BGM method, effectively increasing the local characteristics of the convective-scale weather system and helping to obtain more reasonable initial ensemble members.

\section{c. Comparative analysis on ensemble spread}

Information entropy is used to measure the uncertainty of a perturbation distribution field, and ensemble spread is used to measure the uncertainty of an ensemble prediction system. Ensemble spread represents the degree of divergence among ensemble members, which is one of the important indicators in the investigation of the performance of an ensemble prediction system. The ensemble dispersion in this study refers to the dispersion among the perturbations of the members in the growth phase (i.e., the standard deviation between the perturbation of each ensemble member and the perturbation ensemble average). The calculation formula is as follows:

$$
\begin{aligned}
& \operatorname{Sp}(k, t) \\
& \quad=\sqrt{\frac{1}{m \times n} \sum_{i=1}^{m} \sum_{j=1}^{n}\left\{\frac{1}{N} \sum_{r=1}^{N}\left[X_{r}(i, j, k, t)-\bar{X}(i, j, k, t)\right]^{2}\right\}},
\end{aligned}
$$

where $\bar{X}(i, j, k, t)=(1 / N) \sum_{r=1}^{N} X_{r}(i, j, k, t)$ is the ensemble average of a perturbation variable, $N$ is the number of members in the ensemble prediction system, and $m$ and $n$ are the numbers of the meridional and zonal grids in the study area, respectively. That is, the standard deviation between the perturbation of each ensemble member and the ensemble average is calculated first, and then the regional averages of all grids located at the same level are derived.

Figure 8 shows the evolution of the ensemble spread of each perturbation variable during the growth phase. At the initial time point, the ensemble spread of the traditional BGM and LBGM systems was equal because the two methods generated the same initial 
$\mathrm{U}(\mathrm{m} / \mathrm{s})$

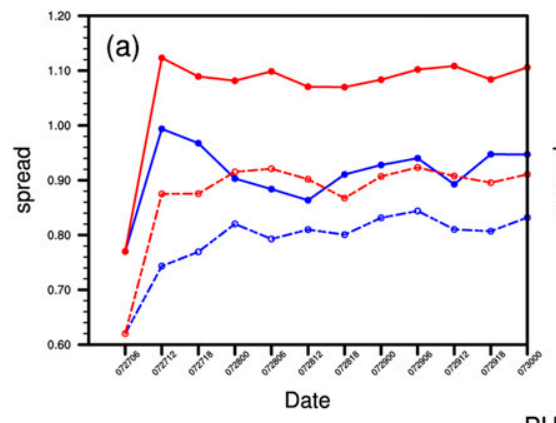

$\mathrm{V}(\mathrm{m} / \mathrm{s})$

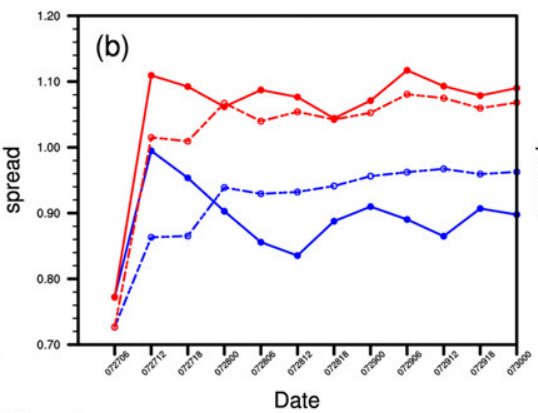

$\mathrm{PH}$ (gpm)

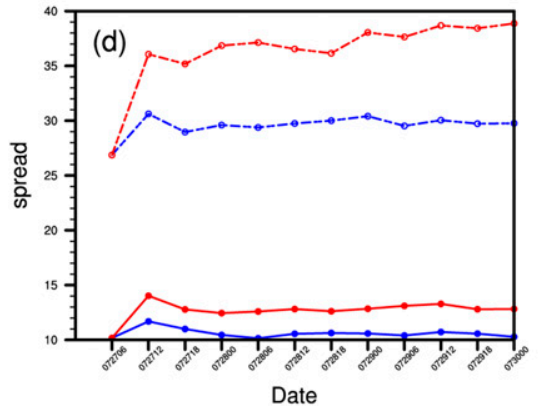

Date $Q(\mathrm{~g} / \mathrm{kg})$
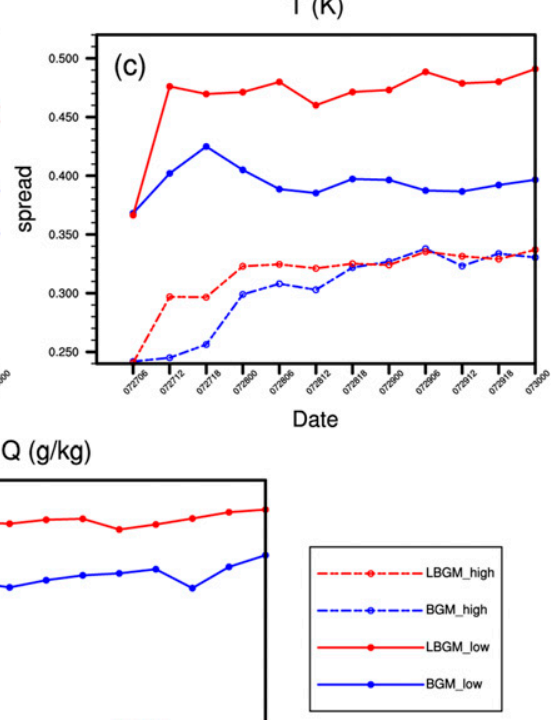

FIG. 8. As in Fig. 7, but for the ensemble spread.

perturbation. After the perturbation grew in the cycles of the WRF model, the ensemble spread increased and remained basically stable. The dispersion degree of each perturbation variable obtained by the LBGM method is greater than that of the traditional BGM method (the red line is located above the blue line of the corresponding level), whether it is at lower or higher layers. For the distribution of ensemble spread at different layers, the analysis was performed with results shown in Fig. 8.

Figure 9 shows the vertical profile of the ensemble spread of the final perturbation variable at 0000 UTC on the 30th day, while the vertical profile of the ensemble spread at the remaining perturbation time points was similar (figure omitted). There is a "double-peak" phenomenon in the ensemble spread of the horizontal perturbation wind field (Figs. 9a,b), that is, a maximum occurs near 850 and $200 \mathrm{hPa}$, and the trough value lies in the middle layer. This may be due to the fact that the lower-layer wind fields are greatly influenced by nearsurface factors, and the higher-layer wind fields have a greater magnitude. For the ensemble spread of the perturbation potential temperature (Fig. 9c), the results of the BGM method do not change significantly in the middle and lower layers, while the LBGM method can significantly increase the ensemble spread of the system in the lower layers of the model. The ensemble spread of the perturbation geopotential (Fig. 9d) increases with height, while the ensemble spread of the water vapor mixing ratio (Fig. 9e) decreases with height, due to the vertical distribution of the magnitude of the perturbation geopotential and water vapor mixing ratio in the atmosphere. This shows that the perturbation results obtained after the two ensemble prediction systems grew in cycles have thermodynamic and kinetic significance. Comparing the results of the two systems, the ensemble spread of all perturbation variables at all layers in the LBGM method is larger than that of the traditional BGM method, which shows that the LBGM method can significantly improve the dispersion of the ensemble prediction system over what is obtained by the traditional BGM method. Therefore, the perturbation of the ensemble members obtained by the LBGM method is more likely to capture the real state of the atmosphere compared to the BGM method.

\section{d. Comparative analysis on prediction RMSE and spread}

This section briefly compares the results of the prediction stages of the two systems. To accurately determine how the LBGM method and the traditional BGM method influence the prediction effects, the perfect model assumptions of Johnson and Wang (2016) were applied. That is, assuming that the model uncertainty is zero, the control prediction of the prediction 

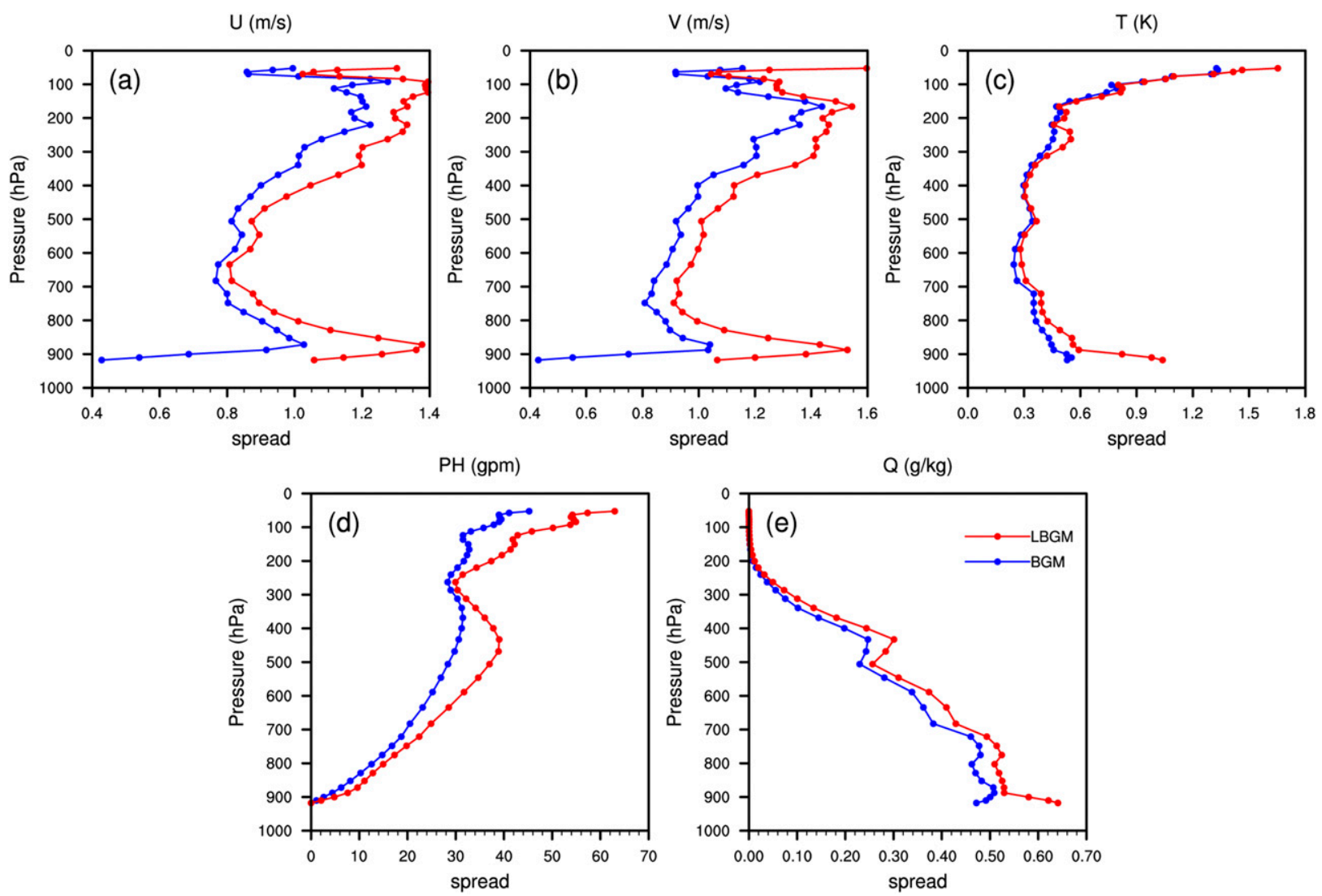

FIG. 9. Vertical profiles of the ensemble spread of the final perturbation variable at 0000 UTC on the 30th day. The line descriptions are the same as in Fig. 7.

system is taken as a true value, and the error introduced by the actual observation data during interpolation is avoided. Thus, the prediction RMSE obtained is only caused by the difference in the growth methods of the two ensemble prediction systems. The calculation formula is as follows:

\section{$\operatorname{RMSE}(k, t)$}

$$
=\sqrt{\frac{1}{m \times n} \sum_{i=1}^{m} \sum_{j=1}^{n}\left[\bar{X}(i, j, k, t)-X_{\mathrm{ctl}}(i, j, k, t)\right]^{2}},
$$

where $X_{\mathrm{ctl}}(i, j, k, t)$ is the result of control prediction and $\bar{X}(i, j, k, t)$ is the same as in Eq. (4). The RMSE and spread between the ensemble average and control prediction are given here to compare the prediction results of the two ensemble prediction systems.

Figure 10 shows the evolution of ensemble spread of perturbation variables in the prediction phase. Generally, the spread in the prediction phase is similar to that in the growth phase, only with greater amplitude in the high dispersion area in the later prediction phase. From the horizontal wind field (Figs. 10a,b), low-level spread is greater than the higher level, which is corresponds to the results of the growth phase. At the same time, the LBGM-produced spread is greater than the traditional BGM-produced one, effectively promoting the ensemble dispersion of the forecast system. It shows that the high-level spread of LBGM is greater than the low-level spread of the traditional BGM method (the red lines are all above the blue lines). This indicates that LBGM has greater improvement on the wind field spread. In terms of potential temperature (Fig. 10c), LBGM produced greater spread than the traditional BGM method. In comparison, high-level spread is smaller than for the low level. In the first half of the prediction phase we obtained similar ensemble spread, while in the second half, LBGM's spread is greater than the traditional BGM's. The spreads of perturbation geopotential height (Fig. 10d) and water vapor mixing ratio (Fig. 10e) are similar to those in the growth phase. The difference is that the spread of the low-level perturbation geopotential height increases more than in the growth phase, though both methods have roughly same spread of the high-level water vapor mixing ratio. Generally, the spreads of all perturbations of LBGM are greater 

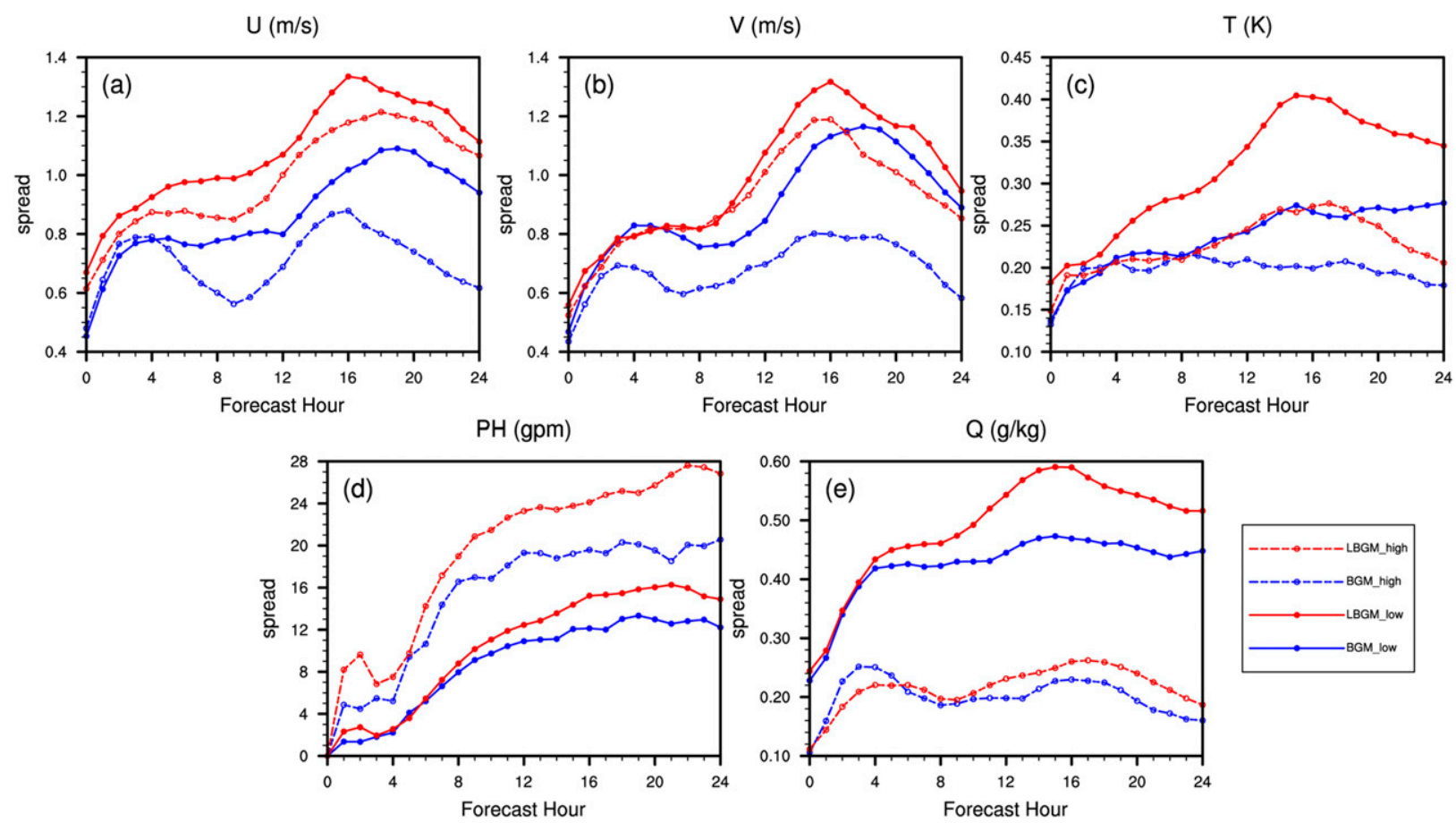

FIG. 10. As in Fig. 7, but for the ensemble spread in the prediction phase.

than of the traditional BGM method. This increases the ensemble dispersion of the forecast system, which becomes as high as in the growth phase.

Figure 11 shows the evolution of RMSE for each perturbation variable. The RMSE of the horizontal wind field (Figs. 11a,b) at the upper layers is less than that of the lower layer, and the RMSE of the LBGM method is smaller than the traditional BGM method (the red line is below the corresponding blue line). With regard to potential temperature (Fig. 11c), in the first half of the prediction, the RMSE of the lower layers is lower than that of the upper layers, and the RMSE of the lower layers in the latter stage is higher than the upper layers. The RMSE of the LBGM method is lower than that of the traditional BGM method (both red lines are located below the blue line) at both the upper and lower layers, indicating that the LBGM method has a significant effect on the prediction of the potential temperature. The geopotential (Fig. 11d) and water vapor mixing ratio (Fig. 11e) are similar to those of the horizontal wind field, except that the RMSE of the geopotential at the lower layers is lower than at the higher layer. This corresponds to the results of the ensemble spread in Figs. 8 and 10. The greater the vertical distribution and variation of variables in the atmosphere, the greater the ensemble spread and the larger the predicted RMSE. That is, there is a certain correspondence between the ensemble spread and the prediction RMSE.
The higher-layer RMSE of the water vapor mixing ratio is comparable in the two methods, which may be the reason why the higher-layer water vapor content is less. In general, the RMSE of each perturbation variable obtained by the LBGM method is basically smaller than that of the corresponding BGM method at the corresponding layer. Therefore, the LBGM method can further reduce the prediction RMSE and improve the prediction effect of the ensemble prediction system.

\section{e. Comparative analysis on observed and predicted precipitation}

To better compare the actual prediction effectiveness between two perturbation methods, the rainfall process brought by the squall line was compared and analyzed in the major period (0700-1100 UTC 30 July) of this convective-scale weather system. Figures $12 \mathrm{a}-\mathrm{d}$ show the evolution of observed hourly accumulated precipitation distribution. We can see the sweeping southward above Jiangsu and Anhui province of the squall line. The precipitation distribution is a zonal band and split into two parts. Figures $12 \mathrm{e}-\mathrm{h}$ and $12 \mathrm{i}-\mathrm{l}$ are the ensemble mean predicted precipitation evolution of the traditional BGM and LBGM methods. Compared to the observation, we know that these two methods can both predict the precipitation generally. But the range was slightly larger and northward. Comparatively, LBGM produces a clearer arched shape of the precipitation 
$\mathrm{U}(\mathrm{m} / \mathrm{s})$

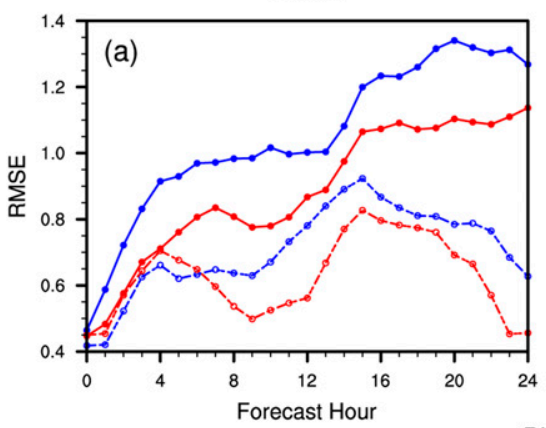

$\mathrm{V}(\mathrm{m} / \mathrm{s})$

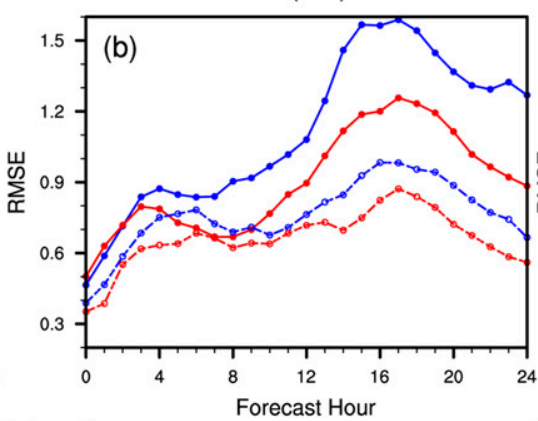

$\mathrm{PH}(\mathrm{gpm})$

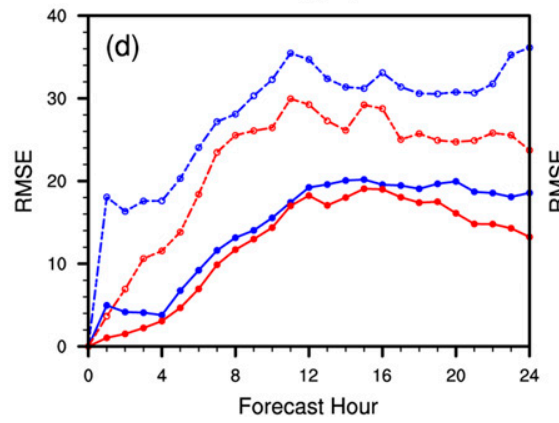

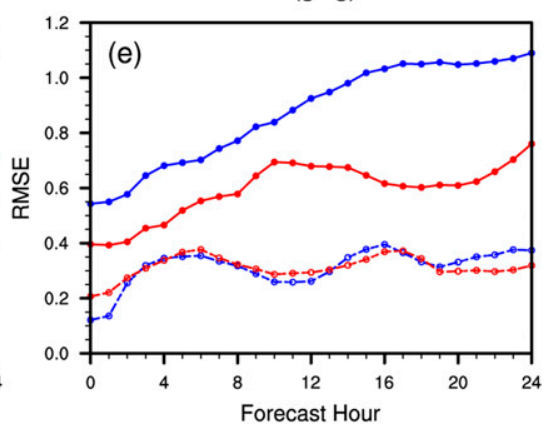

$\mathrm{T}(\mathrm{K})$

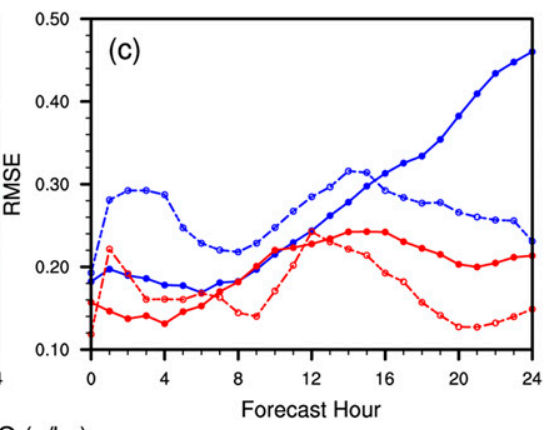

$Q(g / k g)$

FIG. 11. As in Fig. 7, but for the RMSE in the prediction phase.

area than the traditional BGM method, especially in Figs. 12f,g. Meanwhile, the LBGM method provides more local characteristics, and small precipitation centers were ranged tightly. This is more similar to the observation, rather than a single center produced by the traditional BGM method.

For the eastern part of the rainfall area, its characteristics are shown in Figs. 12f, 12g, 12j, and 12k. For the western part, this feature is even more distinctive, as is shown in the black frame. In Fig. 12k, the three precipitation centers produced by the LBGM method correspond to the observation field in Fig. 12c, while the traditional BGM method only produces one center (Fig. 12g). This feature can be seen in the comparison of Figs. 12h and 12l. This further proves that the LBGM method can include the local features of a convective-scale weather system and produce better precipitation prediction than the traditional BGM method.

\section{Conclusions}

In this study, two perturbation generation methods (i.e., the traditional BGM method and a new LBGM method) were used in combination with a strong convective weather process to conduct convection-allowing ensemble prediction experiments. The evolution characteristics of initial perturbations were compared and analyzed with respect to the perturbation morphology distribution, information entropy, and ensemble spread. The prediction RMSEs and spreads of perturbation variables, and the result of predicted precipitation obtained by the two perturbation methods were briefly analyzed, and the evaluation and verification of the LBGM method were performed. The main conclusions are as follows:

1) The LBGM method can not only ensure the overall structure of the perturbation distribution field but also increase the local interaction characteristics of the convective-scale weather system, and the resulting perturbation distribution exhibits characteristics more evident of flow dependence than the traditional BGM method.

2) Information entropy theory can well measure the amount of local information in the perturbation field and quantify the characteristics of the local information of the intuitive perturbation morphology distribution, further indicating that the LBGM method can increase the amount of information of the initial perturbation compared to the traditional BGM method, which helps to obtain more appropriate initial ensemble members.

3) The ensemble spread of each perturbation variable obtained by the LBGM method is larger than that of the traditional BGM method, which can significantly 

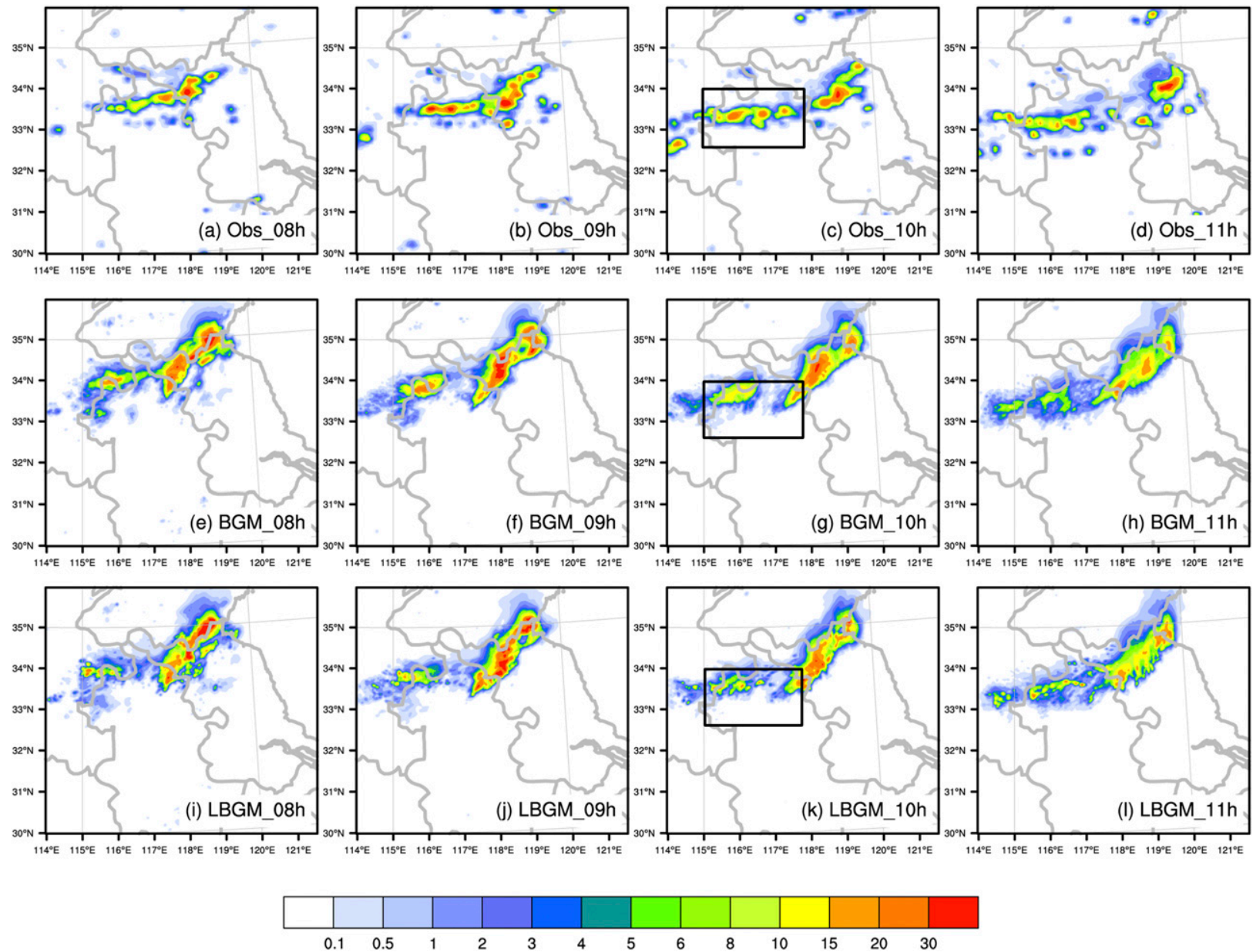

FIG. 12. (a)-(d) Observations and the corresponding (e)-(h) BGM predictions and (i)-(l) LBGM predictions of 8-11-hourly accumulated precipitation $(\mathrm{mm})$ initialized at 0000 UTC 30 July.

improve the dispersion of the ensemble prediction system, thereby solving the problem of insufficient ensemble spread of prediction systems obtained by the traditional BGM method.

4) Compared to the traditional BGM method, the LBGM method can further reduce the prediction RMSE of each perturbation variable, thereby improving the prediction effect of convectionallowing ensemble prediction and the performance of an ensemble prediction system. At the same time, the precipitation distribution predicted by the LBGM method is closer to the observed precipitation than the traditional BGM method, thereby proving the advantage of the LBGM method in actual prediction.

The LBGM method, as an initial perturbation generation method, can increase the local information of perturbation variables and improve the dispersion of the ensemble prediction system, enabling the initial perturbations to have a more definite kinetic significance, which is more likely to capture the real state of the atmosphere. This indicates that the LBGM method has more advantages than the traditional BGM method in convection-allowing ensemble prediction. However, the prediction RMSE reported in this study was based on the assumption of a perfect model, and more other actual observation data are needed to verify the prediction effect. At the same time, suitability tests combined with a large number of cases of severe convective weather in the background of different weather systems are needed in the LBGM method.

Acknowledgments. This paper is jointly supported by the Ministry of Science and Technology of China (2017YFC1501803), the Natural Science Foundation of Nanjing Joint Center of Atmosphere Research (NJCAR2016MS02), and the National Natural Science Foundation of China (NSFC) (Grants 41205073 and 41275012). The data used in this study can be 
downloaded via https://rda.ucar.edu/datasets/ds083.2/ index.html and are available by request to Shenjia $\mathrm{Ma}$ through masj_nj@163.com.

\section{REFERENCES}

Abramov, R., A. Majda, and R. Kleeman, 2005: Information theory and predictability for low-frequency variability. J. Atmos. Sci., 62, 65-87, https://doi.org/10.1175/JAS-3373.1.

Arribas, A., 2005: Test of a poor man's ensemble prediction system for short-range probability forecasting. Mon. Wea. Rev., 133, 1825-1839, https://doi.org/10.1175/MWR2911.1.

Bentzien, S., and P. Friederichs, 2012: Generating and calibrating probabilistic quantitative precipitation forecasts from the high-resolution NWP model COSMO-DE. Wea. Forecasting, 27, 988-1002, https://doi.org/10.1175/WAF-D-11-00101.1.

Bishop, C. H., B. J. Etherton, and S. J. Majumdar, 2001: Adaptive sampling with the ensemble transform Kalman filter. Part I: Theoretical aspects. Mon. Wea. Rev., 129, 420-436, https://doi.org/ 10.1175/1520-0493(2001)129<0420:ASWTET>2.0.CO;2.

Bowler, N. E., A. Arribas, and K. R. Mylne, 2008: The benefits of multianalysis and poor man's ensembles. Mon. Wea. Rev., 136, 4113-4129, https://doi.org/10.1175/2008MWR2381.1.

Buizza, R., and T. N. Palmer, 1995: The singular vector structure of the atmospheric global circulation. J. Atmos. Sci., 52, 1434 1456, https://doi.org/10.1175/1520-0469(1995)052<1434:TSVSOT> 2.0.CO;2.

Chen, C. H., X. Li, H. R. He, J. Xiang, and S. J. Ma, 2018: Algorithm based on local breeding of growing modes for convection-allowing ensemble forecasting. Sci. China Earth Sci., 61, 462-472, https://doi.org/10.1007/s11430-017-9167-5.

Clark, A. J., and Coauthors, 2012: An overview of the 2010 hazardous weather testbed experimental forecast program spring experiment. Bull. Amer. Meteor. Soc., 93, 55-74, https:// doi.org/10.1175/BAMS-D-11-00040.1.

DelSole, T., 2005: Predictability and information theory. Part II: Imperfect forecasts. J. Atmos. Sci., 62, 3368-3381, https:// doi.org/10.1175/JAS3522.1.

Ding, R. Q., J. P. Li, and B. S. Li, 2017: Determining the spectrum of the nonlinear local Lyapunov exponents in a multidimensional chaotic system. Adv. Atmos. Sci., 34, 1027-1034, https:// doi.org/10.1007/s00376-017-7011-8.

Ebert, E. E., 2008: Fuzzy verification of high-resolution gridded forecasts: A review and proposed framework. Meteor. Appl., 15, 51-64, https://doi.org/10.1002/met.25.

Feng, J., R. Q. Ding, D. Q. Liu, and J. P. Li, 2014: The application of nonlinear local Lyapunov vectors to ensemble predictions in the Lorenz systems. J. Atmos. Sci., 71, 3554-3567, https:// doi.org/10.1175/JAS-D-13-0270.1.

Gao, F., J. Z. Min, and F. Y. Kong, 2010: Experiment of the stormscale ensemble forecast based on breeding of growing mode (in Chinese). Plateau Meteor., 29, 429-436.

Harnisch, F., and C. Keil, 2015: Initial conditions for convectivescale ensemble forecasting provided by ensemble data assimilation. Mon. Wea. Rev., 143, 1583-1600, https://doi.org/ 10.1175/MWR-D-14-00209.1.

Hoffman, R. N., and E. Kalnay, 1983: Lagged average forecasting, an alternative to Monte Carlo forecasting. Tellus, 35A, 100 118, https://doi.org/10.3402/tellusa.v35i2.11425.

Houtekamer, P. L., and J. Derome, 1995: Methods for ensemble prediction. Mon. Wea. Rev., 123, 2181-2196, https://doi.org/ 10.1175/1520-0493(1995)123<2181:MFEP>2.0.CO;2.
Jiang, Z. N., M. Mu, and D. H. Wang, 2009: Ensemble prediction experiments using conditional nonlinear optimal perturbation. Sci. China: Earth Sci., 52, 511-518, https://doi.org/ 10.1007/s11430-009-0042-y.

Johnson, A., and X. Wang, 2016: A study of multiscale initial condition perturbation methods for convection-permitting ensemble forecasts. Mon. Wea. Rev., 144, 2579-2604, https:// doi.org/10.1175/MWR-D-16-0056.1.

Jones, T. A., and D. J. Stensrud, 2012: Assimilating AIRS temperature and mixing ratio profiles using an ensemble Kalman filter approach for convective-scale forecasts. Wea. Forecasting, 27, 541-564, https://doi.org/10.1175/WAF-D-11-00090.1.

Kong, F., and Coauthors, 2008: Real-time storm-scale ensemble forecast 2008 spring experiment. 24th Conf. on Severe Local Storms, Savannah, GA, Amer. Meteor. Soc., 12.3, https:// ams.confex.com/ams/24SLS/techprogram/paper_141827.htm.

_ , and Coauthors, 2009: A real-time storm-scale ensemble forecast system: 2009 Spring Experiment. 23rd Conf. on Weather Analysis and Forecasting/19th Conf. Numerical Weather Prediction, Omaha, NE, Amer. Meteor. Soc., 16A.3, https://ams.confex.com/ams/23WAF19NWP/techprogram/ paper_154118.htm.

Kühnlein, C., C. Keil, G. C. Craig, and C. Gebhardt, 2014: The impact of downscaled initial condition perturbations on convective-scale ensemble forecasts of precipitation. Quart. J. Roy. Meteor. Soc., 140, 1552-1562, https://doi.org/10.1002/ qj.2238.

Leith, C. E., 1974: Theoretical skill of Monte Carlo forecasts. Mon. Wea. Rev., 102, 409-418, https://doi.org/10.1175/1520-0493(1974) $102<0409: \mathrm{TSOMCF}>2.0 . \mathrm{CO} ; 2$.

Li, A. B., L. F. Zhang, Q. L. Wang, B. Li, Z. Z. Li, and Y. Q. Wang, 2013: Information theory in nonlinear error growth dynamics and its application to predictability: Taking the Lorenz system as an example. Sci. China: Earth Sci., 56, 1413-1421, https:// doi.org/10.1007/s11430-012-4506-0.

Li, X., H. R. He, C. H. Chen, Z. Q. Miao, and S. G. Bai, 2017: Convection-allowing ensemble forecast based on the breeding growth method and associated optimization of precipitation forecast. J. Meteor. Res., 31, 955-964, https://doi.org/10.1007/ s13351-017-6695-0.

Ma, S. J., C. H. Chen, H. R. He, D. Wu, and C. X. Zhang, 2018a: Assessing the skill of convection-allowing ensemble forecasts of precipitation by optimization of spatial-temporal neighborhoods. Atmosphere, 9, 43, https://doi.org/10.3390/ atmos 9020043.

,,,--- X. Li, and Y. Li, 2018b: Experiment and verification of the convective-scale ensemble forecast based on BGM (in Chinese). Plateau Meteor., 37, 495-504.

Magnusson, L., E. K. Llén, and J. Nycander, 2008: Initial state perturbations in ensemble forecasting. Nonlinear Processes Geophys., 15, 751-759, https://doi.org/10.5194/npg-15-751-2008.

Mu, M., and Z. N. Jiang, 2008: A new approach to the generation of initial perturbations for ensemble prediction: Conditional nonlinear optimal perturbation. Chin. Sci. Bull., 53, 2062-2068, https://link.springer.com/article/10.1007/ s11434-008-0272-y.

Pena, M., and E. Kalnay, 2004: Separating fast and slow modes in coupled chaotic systems. Nonlinear Processes Geophys., 11, 319-327, https://doi.org/10.5194/npg-11-319-2004.

Peralta, C., Z. B. Bouallègue, S. E. Theis, C. Gebhardt, and M. Buchhold, 2012: Accounting for initial condition uncertainties in COSMO-DE-EPS. J. Geophys. Res., 117, D07108, https://doi.org/10.1029/2011JD016581. 
Roberts, N. M., and H. W. Lean, 2008: Scale-selective verification of rainfall accumulations from high-resolution forecasts of convective events. Mon. Wea. Rev., 136, 78-97, https://doi.org/ 10.1175/2007MWR2123.1.

Schwartz, C. S., G. S. Romine, K. R. Smith, and M. L. Weisman, 2014: Characterizing and optimizing precipitation forecasts from a convection-permitting ensemble initialized by a mesoscale ensemble Kalman filter. Wea. Forecasting, 29, 12951318, https://doi.org/10.1175/WAF-D-13-00145.1.

,-- R. A. Sobash, K. R. Fossell, and M. L. Weisman, 2015a: NCAR's experimental real-time convection-allowing ensemble prediction system. Wea. Forecasting, 30,1645-1654, https:// doi.org/10.1175/WAF-D-15-0103.1.

, - M. L. Weisman, R. A. Sobash, K. R. Fossell, K. W. Manning, and S. B. Trier, 2015b: A real-time convectionallowing ensemble prediction system initialized by mesoscale ensemble Kalman filter analyses. Wea. Forecasting, 30, 11581181, https://doi.org/10.1175/WAF-D-15-0013.1.

Shannon, C. E., 1948: A mathematical theory of communication. Bell Syst. Tech. J., 27, 379-423, https://doi.org/10.1002/ j.1538-7305.1948.tb01338.x.

Tennant, W., 2015: Improving initial condition perturbations for MOGREPS-UK. Quart. J. Roy. Meteor. Soc., 141, 2324-2336, https://doi.org/10.1002/qj.2524.

Toth, Z., and E. Kalnay, 1993: Ensemble forecasting at NMC: The generation of perturbations. Bull. Amer. Meteor. Soc., 74, 2317-2330, https://doi.org/10.1175/1520-0477(1993)074<2317: EFANTG $>2.0 . \mathrm{CO} ; 2$.

_- and —_, 1997: Ensemble forecasting at NCEP and the breeding method. Mon. Wea. Rev., 125, 3297-3319, https://doi.org/10.1175/1520-0493(1997)125<3297:EFANAT> 2.0.CO;2.

Wang, X., and C. H. Bishop, 2003: A comparison of breeding and ensemble transform Kalman filter ensemble forecast schemes. J. Atmos. Sci., 60, 1140-1158, https://doi.org/10.1175/ 1520-0469(2003)060<1140:ACOBAE $>2.0 . C O ; 2$.

Wang, Y., and Coauthors, 2011: The central European limited-area ensemble forecasting system: ALADIN-LAEF. Quart. J. Roy. Meteor. Soc., 137, 483-502, https://doi.org/10.1002/qj.751.

_ - S. Tascu, F. Weidle, and K. Schmeisser, 2012: Evaluation of the added value of regional ensemble forecasts on global ensemble forecasts. Wea. Forecasting, 27, 972-987, https:// doi.org/10.1175/WAF-D-11-00102.1.

Wei, M., Z. Toth, R. Wobus, and Y. Zhu, 2008: Initial perturbations based on the ensemble transform (ET) technique in the NCEP global operational forecast system. Tellus, $\mathbf{6 0 A}, 62-79$, https:/ doi.org/10.1111/j.1600-0870.2007.00273.x.

Weidle, F., Y. Wang, and G. Smet, 2016: On the impact of the choice of global ensemble in forcing a regional ensemble system. Wea. Forecasting, 31, 515-530, https://doi.org/10.1175/ WAF-D-15-0102.1.

Weusthoff, T., F. Ament, M. Arpagaus, and M. W. Rotach, 2010: Assessing the benefits of convection-permitting models by neighborhood verification: Examples from MAP D-PHASE. Mon. Wea. Rev., 138, 3418-3433, https://doi.org/10.1175/ 2010MWR3380.1.

Xue, M., and Coauthors, 2007: CAPS real-time storm-scale ensemble and high-resolution forecasts as part of the NOAA Hazardous Weather Testbed 2007 spring experiment. 22nd Conf. on Weather Analysis and Forecasting/18th Conf. on Numerical Weather Prediction, Salt Lake City, UT, Amer. Meteor. Soc, 3B.1, https://ams.confex.com/ams/22WAF18NWP/ techprogram/paper_124587.htm.

Yu, Y. F., and L. F. Zhang, 2005: A study of initial perturbation saturation in ensemble prediction based on the "breeding of growing modes" method. Chin. J. Atmos. Sci., 29, 955-964.

Zhang, F., Y. Weng, Y. H. Kuo, J. S. Whitaker, and B. Xie, 2010: Predicting Typhoon Morakot's catastrophic rainfall with a convection-permitting mesoscale ensemble system. Wea. Forecasting, 25, 1816-1825, https://doi.org/10.1175/2010WAF2222414.1.

Zhang, H. B., J. Chen, X. F. Zhi, and Y. N. Wang, 2015: A comparison of ETKF and downscaling in a regional ensemble prediction system. Atmosphere, 6, 341-360, https://doi.org/ 10.3390/atmos6030341.

—, X. F. Zhi, J. Chen, Z. P. Wu, Y. Xia, and X. R. Zhang, 2017: Achievement of perturbation methods for regional ensemble forecast (in Chinese). Trans. Atmos. Sci., 40, 145-157.

Zhang, L. F., and Y. Luo, 2009: Study of the evolvement of initial errors in ensemble forecast of heavy rains based on BGM (in Chinese). J. Trop. Meteor., 25, 571-575.

Zhuang, X. R., J. Z. Min, Y. C. Cai, and H. N. Zhu, 2016: Convective-scale ensemble prediction experiments under different large-scale forcing with consideration of uncertainties in initial and lateral boundary condition (in Chinese). Acta Meteor. Sin., 74, 244-258.

—_, - - S. Z. Wang, K. Zhou, and Y. C. Cai, 2017: A blending method for storm-scale ensemble forecast and its application to Beijing extreme precipitation event on July 21, 2012. Chin. J. Atmos. Sci., 41, 30-42, https://doi.org/ 10.3878/j.issn.1006-9895.1605.15233. 